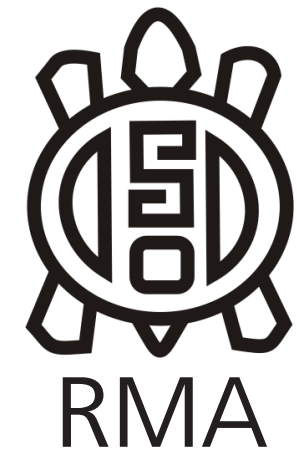

Arqueología

\title{
Prácticas materiales entre los habitantes de La Alumbrera (Puna meridional argentina): contribuciones a partir de los primeros análisis múltiples de microfósiles
}

\author{
Material practices among La Alumbrera inhabitants (Andean \\ Highlands, Catamarca, Argentina): contributions from \\ the first microfossils multiple analyses
}

Alejandra Elías*, Mariana Maloberti**, Verónica Puente***, Daniel Olivera****, Mónica Burgos**** y Julieta Zapatiel******

*Consejo Nacional de Investigaciones Científicas y Técnicas, Instituto Nacional de Antropología y Pensamiento Latinoamericano, Argentina. E-mail: alejandra.elias2@gmail.com

**Instituto de Arqueología y Museo / Facultad de Ciencias Naturales e Instituto Miguel Lillo / Universidad Nacional de Tucumán, Argentina. E-mail: marianamaloberti@yahoo.com.ar

***Laboratorio de Arqueología Regional Bonaerense / Facultad de Humanidades / Universidad Nacional de Mar del Plata, Instituto de Humanidades y Ciencias Sociales / Consejo Nacional de Investigaciones Científicas y Técnicas, Argentina. E-mail: vpuente@mdp.edu.ar ****Facultad de Filosofía y Letras / Universidad de Buenos Aires, Consejo Nacional de Investigaciones Científicas y Técnicas, Instituto Nacional de Antropología y Pensamiento Latinoamericano, Argentina.

E-mail: deolivera@gmail.com

*****Laboratorio de Arqueobotánica / Instituto Superior de Estudios Sociales / Consejo Nacional de Investigaciones Científicas y Técnicas / Universidad Nacional de Tucumán, Argentina. E-mail: kin260882@gmail.com ******Laboratorio de Arqueobotánica / Instituto Superior de Estudios Sociales / Consejo Nacional de Investigaciones Científicas y Técnicas / Universidad Nacional de Tucumán, Argentina. E-mail: julietzap@hotmail.com

\begin{abstract}
Resumen
Este trabajo discute los datos obtenidos mediante análisis múltiples de microfósiles efectuados a la primera vasija completa rescatada estratigráficamente en La Alumbrera, en la microrregión de Antofagasta de la Sierra, Puna Argentina. Junto a la caracterización técnico-morfológica inicial del recipiente, permitieron una aproximación a los modos en que fue usado y manufacturado. Esto contribuye a la documentación diacrónica y sincrónica de las prácticas materiales reproducidas por los habitantes del sitio a fin de compararlas con las de los residentes de otros sectores de la microrregión para avanzar en la comprensión de las complejas y particulares relaciones sociales, políticas, económicas, culturales e identitarias que reprodujeron, negociaron y transformaron, discursiva y no discursivamente, consciente e inconscientemente, luego de ca. 1100 años AP.
\end{abstract}

Palabras clave: Análisis múltiple de microfósiles; Prácticas de manufactura de alfarería; Prácticas de uso de alfarería; Período Tardío del NOA; Puna meridional argentina.

\begin{abstract}
This work discusses the data obtained through multiple microfossils analysis made to the first complete vessel surveyed at La Alumbrera site (micro-region of Antofagasta de la Sierra, Andean Highlands, Catamarca, Argentina) during stratigraphic excavations. Together with the initial technical-morphological characterization of the container, the archaeobotanical analyses allowed an approach to the ways in which it was used and manufactured. This contributes to diachronic and synchronic documentation of material practices reproduced by the inhabitants of the site and compare them with those of the inhabitants of other sectors of the micro-region to understand the complex and particular social, political, economic, cultural and identity relationships that they reproduced, negotiated, and transformed, discursively and not discursively, consciously, and unconsciously, after ca. 1100 years $B P$.
\end{abstract}

Keywords: Multiple microfossils analyze; Pottery manufacturing practices; Pottery use practices; NOA Late Period; Southern Argentine Puna. 


\section{Introducción}

La Alumbrera es un sitio de grandes dimensiones habitado a partir de ca. 1100 años AP (Figura 1). Excavaciones realizadas en una de las estructuras que integran este asentamiento dejaron en evidencia la primera vasija completa rescatada estratigráficamente, la que fue sometida a análisis múltiples de microfósiles. En este trabajo, exponemos y discutimos los datos obtenidos mediante estos estudios, las hipótesis derivadas a partir de ellos respecto a cómo fue utilizado el recipiente a lo largo de su vida e incluimos una caracterización técnicomorfológica preliminar.

La información e interpretaciones presentadas contribuyen a la documentación de la variabilidad de las prácticas materiales de uso y manufactura de alfarería reproducidas por quienes ocuparon La Alumbrera, y, en una escala más amplia, al conocimiento de la diversidad de éstas entre las sociedades antofagasteñas posteriores a ca. 1100 años AP.

\section{El escenario social, político y económico microrregional tardío}

Durante el Período Tardío o de Desarrollos Regionales del NOA (ca. 1100-550 años AP), más específicamente a partir de ca. 700 años AP, las sociedades que habitaron Antofagasta de la Sierra (ANS) experimentaron un proceso de paulatina centralización social, política y económica, caracterizado por el fortalecimiento de grupos orientados a actividades agrícolas de gran escala, con control de los espacios productivos, capacidad de concentrar excedentes y acceso diferencial a recursos de prestigio. En este escenario, La Alumbrera (LA) habría constituido el centro habitacional político, económico y social más relevante de la microrregión y un importante nodo/centro de intercambio y caravaneo. Este sitio se encuentra en el fondo de cuenca, microambiente de la cuenca del río Punilla entre 3400-3550 msnm con sobresalientes posibilidades para el desarrollo de actividades agrícolasganaderas, debido a su topografía abierta y disponibilidad de agua (Figura 1). Corresponde a un conglomerado de amplias dimensiones con características defensivas y compuesto por una gran diversidad de estructuras construidas a partir de la roca de la ladera norte del volcán Antofagasta, sobre la que se emplaza. Acorde con información cronológica relativa y absoluta (Tabla 1), fue ocupado durante los períodos Tardío, Inka (ca. 550-470 años AP) e Histórico. A LA habrían estado vinculados otros asentamientos cumpliendo el rol de áreas especializadas en actividades agrícolas -i.e. Bajo del Coypar II y Campo Cortaderas; Figura 1 (Olivera y Vigliani, 2000/2002; entre otros).

Por su parte, estudios del arte rupestre sugirieron que, en este escenario, las familias pastoras de los sectores intermedios de las quebradas de Las Pitas y Miriguaca (Figura 1) se integraron a las nuevas formas económicas y sociales operando, como lo venían haciendo desde el primer milenio de la Era, a modo de unidades básicas de producción del componente pastoril, y continuaron reproduciendo sus idiosincrasias tradicionales, distintivas de las de sus coetáneos del fondo de cuenca (Cohen, 2014; Elías, 2010; Elías y Escola, 2018; Escola, Elías, Gasparotti y Sentinelli, 2015; Martel y Aschero, 2007; Pérez y Gasparotti, 2016; Puente, Plá e Invernizzi, 2017; entre otros).

Estos antecedentes nos alentaron a continuar avanzando en la comprensión de la coexistencia de distintos grupos en ANS y sus complejas relaciones sociales, políticas y económicas luego de ca. 1100 años AP (Elías, 2018). La conformación de grupos, límites sociales e identidades son aspectos de la práctica social, constantemente construidos y negociados por las personas, consciente e inconscientemente, discursiva y no discursivamente. En sus prácticas materiales, en el uso y creación cotidiana de su cultura material, los sujetos reproducen sus disposiciones y límites culturales e identitarios. Los modos y formas de hacer, procesar, consumir o usar distintos materiales y objetos (tan mundanos como artefactos líticos, cerámica no decorada, fauna, etc.) son loci claves donde se producen, reiteran y transforman los significados

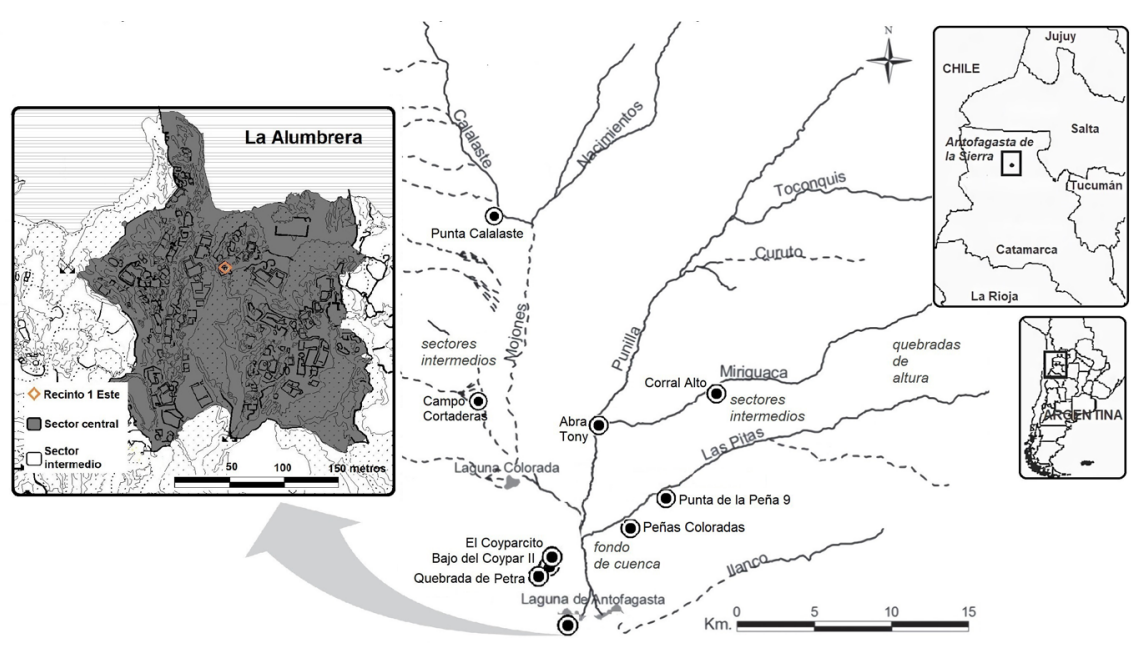

ISSN 1852-060X (impreso) / ISSN 1852-4826 (electrónico)
Figura 1. Microrregión de Antofagasta de la Sierra y plano de La Alumbrera

Figure 1. Antofagasta de la Sierra microregion and La Alumbrera map 
sociales (Pauketat, 2001; Stark, 1998; entre otros). En consecuencia, estudiar la conformación de grupos sociales y de las relaciones sociales, políticas y económicas que reprodujeron entre ellos exige documentar la variabilidad a lo largo del tiempo y el espacio de sus prácticas materiales, considerando contextos diversos y múltiples escalas de análisis (Pauketat, 2001).

Planteamos contribuir a este objetivo aproximándonos comparativamente a la cultura material de los habitantes de LA y del sitio contemporáneo de Punta Calalaste (PCa), emplazado en los sectores intermedios occidentales (Figura 1), concentrándonos en ampliar los muy escasos y/o ausentes contextos estratigráficos y en estudiar los diversos vestigios rescatados. En estos asentamientos disponemos de evidencias arqueológicas principalmente obtenidas en recolecciones de superficie, cuyo estudio produjo interesantes interpretaciones, aunque de acotada resolución cronológica (Elías, 2010; Olivera et al., 2008; Pérez y Gasparotti, 2016; Salminci, 2015; entre otros). De este modo, buscamos aprehender, en forma cronológicamente más específica y estadísticamente más significativa, la diversidad de las prácticas de manufactura y uso de artefactos líticos y cerámicos, de aprovechamiento de fauna, y de consumo de vegetales entre los habitantes de ambos asentamientos (Elías, 2018).

En el marco de estas investigaciones efectuamos los análisis múltiples de microfósiles (sensu Coil, Korstanje, Archer y Hastorf 2003) de la vasija rescatada en uno de los recintos de LA. Inicialmente, fueron realizados con la expectativa de avanzar en la documentación de las prácticas de consumo y post-colecta (sensu Capparelli y Lema, 2010) de recursos vegetales que integraron la dieta de los habitantes del asentamiento y del fondo de cuenca, sobre las que disponemos de escasa información (Killian, Grant, Escola, Panarello y Olivera, 2016; Oliszewski y Olivera, 2009). No obstante, como veremos más adelante, los datos alcanzados no contribuyeron a este fin', aunque sí posibilitaron la formulación de hipótesis relativas al empleo del recipiente.

\section{Contexto de rescate, obtención, procesamiento y análisis de muestras}

La vasija objeto de análisis procede del Recinto 1 Este B, de planta cuadrangular y conectado por medio de una abertura en su muro Oeste al Recinto 1 Este A, de forma rectangular, mayor superficie y con una entrada en su pared norte, único acceso a ambos. Ambas estructuras y una construcción circular inmediatamente al noroeste de ellas - probablemente destinada a actividades funerarias y/o de almacenaje- conforman el Conjunto Arquitectónico

1 Contamos con otras muestras (i.e. sedimentos de capas/niveles y rasgos estratigráficos, de raspado en húmedo y seco en artefactos de molienda) cuyo estudio, mediante diversos análisis (por ej. de microfósiles, de macrorrestos botánicos), posibilitará avanzar en este objetivo (Elías, 2018)
1 Este, delimitado por un muro de piedra de trazado irregular, excepto en su flanco sur donde se levanta la colada basáltica (Figura 2). El recipiente fue relevado en posición, durante la intervención estratigráfica efectuada en el sector sudeste del Recinto 1 Este B. Se hallaba hacia el final de la capa 3, incorporado en los sedimentos de una posible estructura de fogón y con su base apoyada en la capa 4 caracterizada por sedimentos rojizos arenoarcillosos asociados muy posiblemente con rubefacción. Caben mencionar, en términos generales al hallarse aún en proceso de análisis, otras evidencias relevadas: restos de arqueofauna (astillas y huesos enteros), instrumentos, desechos de talla, núcleos y ecofactos líticos (entre ellos una punta de proyectil de obsidiana), escasos tiestos no decorados (muchos de pasta gruesa), pocos restos vegetales y guano (Elías, 2018). No contamos todavía con información cronológica absoluta directamente asociada, aunque sí con un fechado radiocarbónico obtenido en un sondeo realizado en el ángulo noroeste de la estructura (Tabla 1).

El recipiente no presenta decoración y se encuentra tiznado. Siguiendo los criterios establecidos por Shepard (1956) y Balfet, Fauvet y Monzón (1992), su forma es restringida simple, con contorno simple, cuerpo subglobular, base cóncava, borde invertido y labio irregular, a $2 \mathrm{~cm}$ del cual presenta un pequeño botónmamelón. Posee pasta de aspecto grueso, la cocción es oxidante incompleta y registra irregularidades superficiales características de la técnica de manufactura por rollos. Su superficie externa recibió un tratamiento de alisado que dejó marcas en distintas direcciones y seguramente facilitó un buen agarre, mientras que la interna fue objeto de un alisado más fino. Sus dimensiones son considerables: alto de $29,5 \mathrm{~cm}$, diámetro máximo de $36 \mathrm{~cm}$, diámetro de boca de $31,5 \mathrm{~cm}$ y paredes tendientes a gruesas, registrando $1 \mathrm{~cm}$ de espesor en el sector del labio (Elías, 2018; Puente, 2020).

A continuación, detallamos los procedimientos seguidos en la obtención, procesamiento y análisis múltiple de microfósiles de las muestras, aclarando que para la concreción de los dos últimos pasos se contrataron los servicios del Laboratorio de Arqueobotánica del Instituto de Arqueología y Museo -IAM- (Instituto Superior de Estudios Sociales / Consejo Nacional de Investigaciones Científicas y Técnicas / Universidad Nacional de Tucumán).

Una muestra corresponde a las matrices sedimentarias que contenían la pieza (capas 3 y 4). Durante su obtención, con el objetivo de minimizar contaminaciones, se siguieron los protocolos propuestos por Pearsall (2000 [1989]) y Piperno (1988). Posteriormente, para recuperar los microfósiles se procedió a procesar, debido a nuestro acotado presupuesto, sólo la muestra de la capa 4, de acuerdo con los protocolos propuestos por Coil et al. (2003) y Korstanje (2005), los que aseguran bajo impacto químico y la extracción de diferentes clases de 


\begin{tabular}{|c|c|c|c|c|c|c|}
\hline Recinto & Contexto & Nivel/Capa & Código lab. & $\begin{array}{l}\text { Tipo de } \\
\text { muestra }\end{array}$ & $\begin{array}{c}\text { C14 años } \\
\text { AP }\end{array}$ & Referencias \\
\hline $\begin{array}{l}\text { Tumba } \\
\text { colectiva }\end{array}$ & tumba removida & sin datos & sin datos & $\begin{array}{l}\text { restos óseos } \\
\text { humanos }\end{array}$ & $210 \pm 70$ & $\begin{array}{c}\text { Olivera y Vigliani } \\
(2000 / 2002)\end{array}$ \\
\hline 1 Este $b$ & $\begin{array}{c}\text { capa de cenizas y } \\
\text { carbones }\end{array}$ & $2\left(2^{\circ}\right)$ & AA82552 & $\begin{array}{l}\text { carbón } \\
\text { vegetal }\end{array}$ & $916 \pm 50$ & \multirow{5}{*}{$\begin{array}{c}\text { Elías (2010); Elías et } \\
\text { al. 2015; Olivera et } \\
\text { al. (2008) }\end{array}$} \\
\hline Tumba & tumba huaqueada & Superficie & AA82550 & $\begin{array}{c}\text { madera } \\
\text { (tarabita) }\end{array}$ & $534 \pm 59$ & \\
\hline 1 Oeste & $\begin{array}{c}\text { capa con espículas de } \\
\text { carbón y otros restos } \\
\text { arqueológicos }\end{array}$ & 1 & AA78543 & $\begin{array}{l}\text { carbón } \\
\text { vegetal }\end{array}$ & $981 \pm 39$ & \\
\hline \multirow{2}{*}{2 Oeste } & $\begin{array}{c}\text { concentración } \\
\text { carbonosa }\end{array}$ & 2 & AA82551 & $\begin{array}{l}\text { carbón } \\
\text { vegetal }\end{array}$ & $1007 \pm 50$ & \\
\hline & $\begin{array}{l}\text { concentración } \\
\text { carbonosa }\end{array}$ & 3 & AA78542 & $\begin{array}{l}\text { carbón } \\
\text { vegetal }\end{array}$ & $536 \pm 42$ & \\
\hline
\end{tabular}

Tabla 1. Fechados radiocarbónicos de La Alumbrera

Table 1. Radiocarbon dating from La Alumbrera microrrestos.

Las muestras del interior de la vasija fueron obtenidas siguiendo la metodología descripta por Babot (2004). Primero, se seleccionaron los sectores del recipiente a ser muestreados, priorizando aquellos que favorecen al entrampamiento de microrrestos (por ej. irregularidades de la superficie) y/o con presencia de residuos macroscópicos. El muestreo se efectuó en húmedo y seco, con control de tiempo y área, en el borde, cuerpo y base. Extrajimos seis (6) muestras secas y tres (3) muestras húmedas debidamente rotuladas acorde al área de obtención. De estas, por las razones presupuestarias referidas, solo pudimos analizar tres (3), azarosamente elegidas, dos (2)
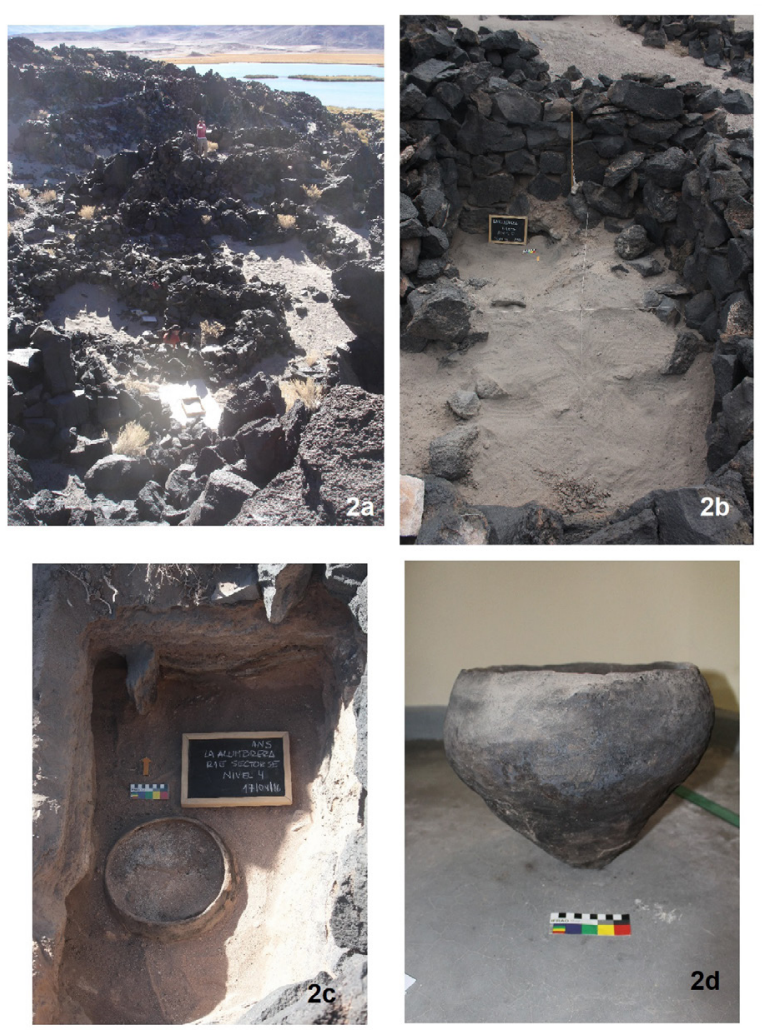

Figura 2. 2a. Conjunto Arquitectónico 1 Este; 2b. Recinto 1 Este B; 2c. vasija en contexto; 2d. vasija. Fotos: Elías (2010, 2018)

Figure 2. 2a. Architectural Assemblage 1 east; $2 b$. Enclosure 1 East B; 2c. vessel in context; 2c. vessel. Photos: Elías (2010, 2018) secas procedentes del borde y del cuerpo del recipiente, y una (1) húmeda de su base.

A fin de posibilitar la rotación controlada de los microfósiles para su descripción tridimensional, todas las muestras fueron montadas en aceite de inmersión y cubiertas por un cubreobjetos sellado con esmalte de uñas incoloro. El análisis se realizó con microscopio petrográfico con luz transmitida y polarizador a un aumento de 400x, empleando la placa de cuarzo para caracterizar principalmente granos de almidón y diferenciarlos de las esferulitas.

La cuantificación y determinación se efectuó contabilizando el $100 \%$ de microrrestos en la totalidad del cubreobjetos. Para su asignación taxonómica se consideraron diferentes publicaciones (Bertoldi de Pomar, 1975; Bozarth, 1992; Fredlund y Tieszen, 1994; Twiss, 1992; Zucol, 1996; entre otros), colecciones de referencia -especialmente la colección de microfósiles de plantas económicas surandinas de Korstanje y Babot (2007)- y bases de datos institucionales (p. ej. la de la Universidad de Missouri). Además, se recurrió a las denominaciones de los códigos ICPN 1.0 (Código Internacional de Nomenclatura para Fitolitos) y ICSN (Código Internacional de Nomenclatura para Almidones). En tanto, para la interpretación taxonómica, anatómica y tafonómica de los microrrestos adheridos a la superficie interna del recipiente se siguieron los aportes de Babot (2004).

\section{Resultados}

Empezaremos exponiendo las tendencias registradas en el interior del recipiente (Figuras 3 y 4). Cabe aclarar que, si bien estas son evaluadas conjuntamente, las muestras de las que se obtuvieron fueron procesadas y analizadas en forma discriminada respetando los distintos sectores de los que fueron extraídas. Pese a que todas remiten al contenido de la vasija, el raspado de diferentes zonas garantiza la recuperación tanto de microfósiles adheridos a su borde y cuerpo, consecuencia de residuos sobrenadantes, como de otros que podrían haberse acumulado por decantación en su base.

Los morfotipos más abundantes entre los fitolitos corresponden al grupo de los elongados, de difícil 
asignación taxonómica por su ubicuidad en diferentes familias botánicas. No obstante, se determinaron taxonómicamente fitolitos asignables a la familia Asteraceae (principalmente placas opacas perforadas) y elevadas frecuencias de silicofitolitos de Poaceae (gramíneas), estos últimos esperables tanto por la alta productividad de fitolitos de esta familia como por las características ambientales microrregionales². Entre las Poaceae es clara la abundancia de las subfamilias Pooidea, Arundinoideae y Stipoideae, mientras que Panicoideae aparece escasamente representada a través, por ejemplo, de morfotipos en forma de cruz psilate o tetralobado variantes 5 y 6 , no diagnósticos de Zea mays (Piperno, 2006). Entre los géneros de Poaceae (subfamilia Stipoideae), reportamos silicofitolitos de Stipa sp. (bilobado psilate tipo Stipa). En lo que refiere a las representaciones anatómicas, se determinaron pelos silicificados afines a los descriptos en la epidermis y sub-epidermis de Poaceae y entre las células cortas silicificadas formas psilate, rondel, acicular y escutiforme observadas en sus hojas, con énfasis en las glumas y lemmas de inflorescencias. Se registraron, también, numerosas células articuladas o espodogramas, mayormente termoalterados. La comparación de algunos de estos con preparados de la colección de referencia del Laboratorio de Arqueobotánica del IAM, permitió reconocer similitudes taxonómicas con los descriptos para el género Cortaderia sp. y Stipa sp. En lo que refiere a los calcifitolitos, debido a su difícil asignación taxonómica, podrían proceder de numerosas familias botánicas. Por su parte, los palinomorfos se hallan representados por granos de polen e hifas y esporas de hongos, posiblemente asociadas a procesos de putrefacción. Entre los primeros, un dato extralocal lo constituyen los de tipo Alnus sp. -"aliso del cerro" (Betulaceae), de dispersión anemófila, transportados a grandes distanciasy otro grano de polen 2-porado (sin identificar). Otros microrrestos relevados son: microcarbones, cristales de calcio, diatomeas (Basillariophytaceae), crisofíceas (Crysophyceae) y espículas de espongiarios asociados probablemente a cuerpos de agua dulce. Los primeros podrían remitir a la asociación de la pieza a eventos de quema, lo que se refuerza por la presencia de silicofitolitos con signos de termoalteración (sensu Parr 2006) y las crisofíceas son posibles indicadores de ambientes con disponibilidad de Nitrógeno (Binford et al. 1996) lo cual puede interpretarse como resultado de la acumulación de residuos orgánicos. En tanto, entre las diatomeas, se registró, recurriendo a Iconographia Diatomologica en Diatoms of the Andes, gran diversidad de especies y/o géneros en todas las muestras, reportándose ejemplares sueltos, así como, en elevadas frecuencias, otros agrupados en filamentos o colonias. Finalmente, cabe

2 En función de sus bajas temperaturas y escasas precipitaciones, la vegetación de la Puna meridional argentina corresponde a la Provincia Puneña del Dominio Andino-Patagónico, caracterizada por el dominio de la estepa arbustiva y presencia de estepas halófila y herbácea, así como de vegas o depresiones donde se acumula el agua de vertiente y crecen gramíneas (Cabrera, 1976). señalar que no se contabilizaron granos de almidón, anillos de celulosa ni esferulitas.

El conjunto de microfósiles de la muestra de sedimento de la capa 4 (Figuras 3 y 4) se acerca al del interior de la vasija por la presencia de crisofíceas y espodogramas y las elevadas representaciones de diatomeas articuladas y en colonias, junto a la ausencia de anillos de celulosa, esferulitas y almidones. No obstante, evidencia también algunas diferencias: 1) presencia de morfotipos de gramíneas no observados en el interior del recipiente
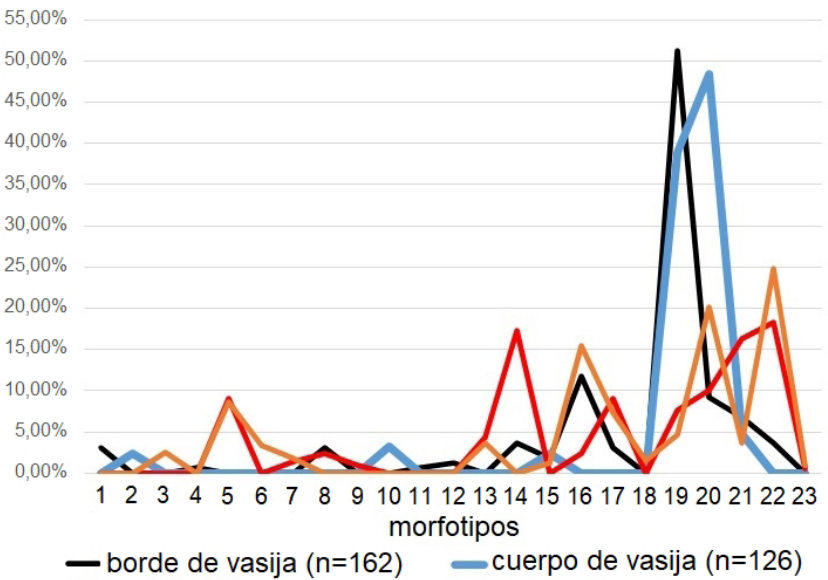

- base vasiia $(n=208)$ - muestras de sedimento $(n=323)$

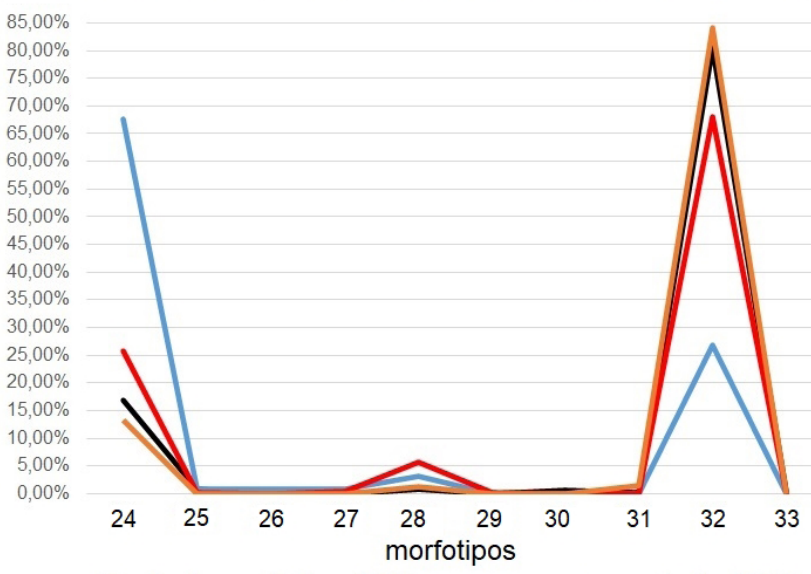

-borde de vasija ( $n=1621)$-cuerpo de vasija $(n=127)$

-base vasija $(n=556)$-muestra de sedimento $(n=1281)$

Referencias: 1) silicofitolito célula corta bilobado tipo Stipa; 2) silicofitolito célula corta bilobado; 3) silicofitolito célula corta bilobado psilate; 4) silicofitolito célula corta cruz var. 5 y 6 ; 5) silicofitolito célula corta psilate; 6) silicofitolito célula corta crenate; 7) silicofitolito célula corta rondel; 8) silicofitolito célula corta escutiforme; 9) silicofitolito célula corta acicular; 10) silicofitolito célula corta irregular; 11) silicofitolito célula buliforme cuneiforme; 12) silicofitolito célula poliédrica; 13) placa perforada Asteraceae; 14) silicofitolito célula larga sinuate; 15) silicofitolito célula larga papillate; 16) silicofitolito célula larga equinate; 17) pelo silicificado; 18) traqueida cilíndrica silicificada; 19) calcifitolito; 20) agregados de cristales con oclusiones: 21) fraqmento veqetal silicificado; 22)

Figura 3. Frecuencias relativas de fitolitos (arriba) y otros microfósiles (abajo)

Figure 3. Relative frequencies of phytoliths (above) and other microfossils (below) 
Figura 4. Conjunto de microfósiles identificados en muestras del interior de la vasija y muestra de sedimento de capa 4 agrupados en silicofitolitos sueltos y articulados, protistas y esporomorfos

Figure 4. Set of microfossils identified in samples from the interior of the vessel and sediment sample from layer 4 grouped among loose and articulated silicophytolites, protist and sporomorphs

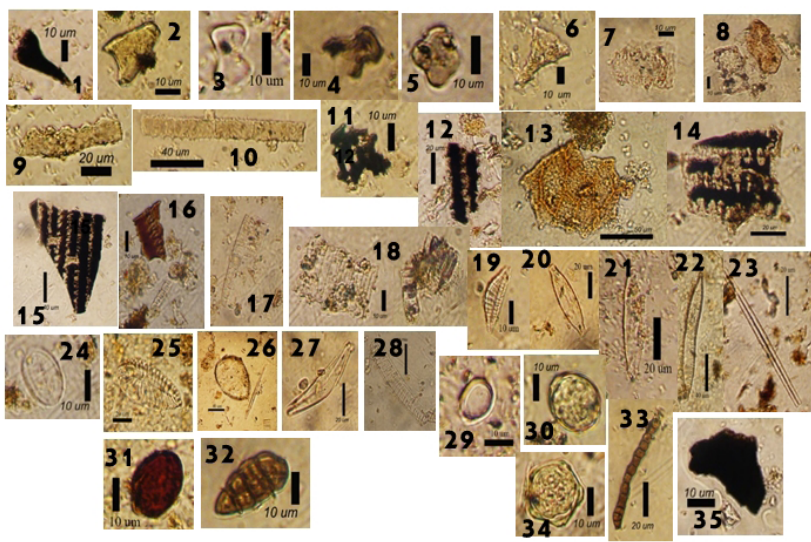

Referencias: Silicofitolitos sueltos y articulados: 1) pelo, 2) escutiforme; Células cortas: 3) bilobado psilate, 4) bilobado tipo Stipa, 5) cruz var. 5 y 6, 6) buliforme cuneiforme, 7 y 8) crenate y equinate respectivamente; células largas: 9 y 10) elongados sinuate y equinate, respectivamente, 11) placa perforada Asteraceae; Células articuladas: 12) papillate, 13) poliedricas, 14) Stipa sp., 15) Cortaderia sp.,16) crenate.

Diatomeas: 17) fragmento, 18) colonias, 19) Rhompalodia aff. gibba, 20) Stauroneis aff. Atacamae, 21) Nitzschia sp., 22) Hantzchia sp., 23) Fragilaria sp., 24) Cocconeis sp., 25) Surirella aff. sella, 26) Surirella aff. fortii, 27) Encyonema sp., 28) Denticula aff. thermalis., 29) estatocisto de Crysophyceae. Fungi: 30) Glomus sp., 31 y 32) esporas, 33) hifa.

Otros: 34) grano de polen Alnus sp, 35) microcarbón

(por ej. células cortas bilobado psilate y crenate) y ausencia de otros sí identificados en él (por ej. células cortas escutiformes, irregulares, aciculares, bilobado tipo Stipa, cruz var. 5 y 6, buliforme cuneiforme, poliédrica y larga sinuate); 2) baja frecuencia de calcifitolitos, lo que puede entenderse considerando la fragilidad de estos microrrestos en los suelos (Babot, 2004); y 3) menor porcentaje de microcarbones y esporas de hongo (Fungi), además de ausencia de esporas de hongo Glomus sp., hifas de hongo, espículas de esponjas, sin dejar de mencionar los granos de polen.

\section{Discusión y conclusiones}

Los resultados alcanzados contribuyen a aproximarnos a algunos de los modos en que la vasija fue utilizada a lo largo de su historia de vida. Inicialmente, la identificación entre los microfósiles de frecuencias elevadas de microcarbones y silicofitolitos quemados lleva a plantear que el recipiente estuvo asociado a eventos de quema, lo que es claramente avalado por su vinculación a un fogón, así como por la presencia de manchas de hollín o tiznado en su superficie externa y de adherencias en el sector cercano al borde probablemente generadas por la acción de un fuego envolvente (Puente, 2020).
Además, el hallazgo de espodogramas termoalterados taxonómicamente afines a Cortaderia sp. y Stipa sp. posibilita considerar el empleo de alguno de estos géneros o de ambos a modo de combustible, pequeño aporte que se suma a los escasos antecedentes disponibles sobre las prácticas de empleo y procesamiento de vegetales no integrados a la dieta de los habitantes de LA (Elías, Marconetto y Olivera, 2015).

En segundo lugar, la sobresaliente representación de microfósiles indicadores de ambientes de elevada humedad en las muestras alienta a hipotetizar que la vasija habría sido empleada como contenedor de líquidos en el espacio del Recinto 1 Este B. Estos indicadores refieren al diverso y abundante registro diatomológico, a las algas Crysophyceae (crisofitas) y a los espodogramas (esqueletos silíceos de células largas y cortas articuladas), así como a las espículas de esponjas y células buliformes cuneiformes observadas sólo en el interior del recipiente. La turgencia de las últimas -en el sentido biológico y químico del término- apoya, asimismo, una condición ambiental favorablemente húmeda dentro de la pieza. Corresponde aclarar que es poco probable que las diatomeas identificadas en las muestras obtenidas en el interior del recipiente provengan del agua incorporada durante su fabricación, ya que, de ser así, deberían observarse altas frecuencias de frústulos de diatomeas fragmentados producto del amasado de la arcilla. Por el contrario, estas se hallan mayormente enteras, articuladas y conformando colonias. Antes de continuar, nos interesa insistir en que las interpretaciones derivadas de los microfósiles identificados en las muestras, incluidas la del interior de la vasija, parten de un análisis llevado adelante desde una aproximación múltiple. De este modo, siguiendo la base interpretativa propuesta por Coil et al. (2003) y Korstanje (2005), ponderamos no solo la presencia o ausencia de un morfotipo en específico, sino principalmente la composición integral de los conjuntos de microrrestos.

En tercer lugar, debemos mencionar que, durante los primeros acercamientos a las muestras, se registró gran cantidad de microfósiles aglomerados de diferente naturaleza (microcarbones, silicofitolitos, calcifitolitos, diatomeas, etc.), lo que exigió, a fin de desagregarlos, procesarlas con mortero y pistilo y deflocularlas mediante la aplicación de Hexametafosfato de Sodio al 5\% durante 12 hs. Aglomeraciones de microrrestos similares fueron observadas en contenedores cerámicos de otras regiones, llevando a realizarles análisis de cromatografía gaseosa con espectrometría de masas y, así, a reportar ceras y aceites de origen vegetal (Maloberti, 2019; Maloberti et al., 2018). Concretaremos a futuro dichos estudios en la pieza de LA a fin de determinar la presencia o no de mezclas complejas orgánicas (por ej. ácidos, aceites, etc.). Paralelamente, estos posibilitarán avanzar en una mejor comprensión de su uso contribuyendo, por ejemplo, a evaluar si contuvo restos orgánicos no detectables desde 
los microrrestos.

Antes de finalizar, nos interesa exponer algunas consideraciones respecto al registro en otras ocupaciones posteriores a ca. 1100 años AP del fondo de cuenca y de las quebradas de Miriguaca y Las Pitas de piezas similares en algunos atributos morfo-tecnológicos a la del Recinto 1 Este B. Una es "una olla periforme, negra, de aspecto ordinario y rústico" (Ambrosetti, 1906, p. 29) hallada por G. Gerling en un sepulcro en cista circular con techo en falsa bóveda próximo al sitio del fondo de cuenca de El Coyparcito (Figura 1). Otra se trata de una porción basal con paredes con espesor promedio de 0,92 cm, base cóncava-convexa, forma subglobular y un diámetro máximo estimado de $40 \mathrm{~cm}$, relevada boca abajo contra uno de los muros de una estructura de Corral Alto (CA), asentamiento de la quebrada de Miriguaca (ver forma reconstruida 'b' en Figura 2 de Gasparotti y Haros, 2015). Estudios de microfósiles identificaron en ella bajas frecuencias de diatomeas, abundantes microcarbones, silicofitolitos y granos de almidón afines a Zea mays (enteros y fracturados y con evidencias de rostizado o tostado), drusas y granos de almidón relacionados a tubérculos (i.e. Solanum sp.), y almidones de los géneros Phaseolus, Prosopis y Chenopodium, conforme a lo cual se propuso que fue empleada para el almacenaje o cocción de vegetales diversos (Gasparotti y Haros, 2015). Una tercera pieza, con borde invertido, base cónica y contorno globular, fue encontrada entera y depositada también boca abajo en un rincón de uno de los recintos de Punta de la Peña 9 (PP9) en la quebrada de Las Pitas (ver Figura 1 de Cohen, 2005). Queda referir dos fragmentos relevados en Peñas Coloradas 3 cumbre (PC3C; Figura 1), también en la última quebrada, asociados a prácticas de consumo y producción de alimentos y otros bienes en un espacio ritual (Cohen, 2014). Uno corresponde a la porción basal cónica de una vasija con paredes de espesores entre 1,6-2,4 cm, mientras que el otro, más grande, con un pequeño apéndice adyacente al borde y paredes con espesores entre 0,8-1,4 cm, formó parte de un recipiente con diámetro de boca estimado de 26 $\mathrm{cm}$ (Puente et al., 2017). Los cuatro últimos ejemplares presentan evidencias de haber sido sometidos al fuego y sus paredes levantadas mediante la técnica de rollos y alisadas en sus superficies.

Ahora bien, a partir de lo mencionado en el párrafo anterior se deducen nuevas similitudes entre los ejemplares y también variaciones. Una de las primeras es que la olla de LA y los recipientes de CA, PP9 y PC3C presentan todos evidencias de haber sido utilizados en eventos de quema. Otra es el hallazgo de los ejemplares de los tres primeros sitios en espacios domésticosresidenciales. Respecto a las variaciones, sobresale el importante registro de diatomeas y la ausencia de maíz, papa y poroto en la vasija de LA, tendencias relacionadas probablemente con el uso particular del que fue objeto y que la distancian del recipiente de CA. En este las diatomeas presentan bajas frecuencias y sí fueron determinados microfósiles asociados con los recursos vegetales referidos -cabe mencionar, asimismo, que restos de poroto fueron relevados en PC3C (Cohen, 2014). Otra disparidad refiere al hallazgo del ejemplar de LA en posición de uso, boca arriba y tapado con lajas (Elías, 2018), en tanto que los contenedores de PP9 y CA fueron hallados boca abajo en posición de guardado. Finalmente, destaca que mientras que las bases de las piezas de PP9 y PC3c presentan formas cónicas, las de los recipientes de LA y CA son cóncavas. Esto podría vincularse a requerimientos funcionales, aunque no descartamos su asociación con la reproducción de prácticas materiales comunes entre los habitantes del fondo de cuenca y de la quebrada de Miriguaca. Al respecto, es interesante señalar que los estudios comparativos de los conjuntos artefactuales líticos y cerámicos procedentes de CA, LA y BCII han tendido a resaltar las discrepancias técnicomorfológica entre ellos para concluir a partir de ellas que los habitantes de la quebrada de Miriguaca, al igual que los de la quebrada de Las Pitas, continuaron reproduciendo disposiciones culturales practicadas por sus ancestros formativos, incluso en el escenario tardío de centralización y fortalecimiento en el fondo de cuenca de grupos con creciente control cultural, social, político y económico (Elías, 2010; Pérez y Gasparotti, 2016). Sin embargo, las afinidades formales entre las bases de los recipientes de LA y CA se suman a otras señaladas entre las evidencias líticas de estos sitios que llevaron a proponer que quienes habitaron la quebrada de Miriguaca luego de ca. 1100 años AP no sólo continuaron reproduciendo sus prácticas materiales tradicionales, sino que también las reactualizaron y transformaron, incorporando otras reproducidas por sus coetáneos del fondo de cuenca (Elías, 2018; Elías y Escola, 2018).

Para concluir nos interesa mencionar que será necesario seguir avanzando en el estudio y comprensión de las prácticas materiales asociadas a la manufactura y uso de la vasija de LA y de los específicos contextos materiales, funcionales, sociales, identitarios y económicos en las que fueron reproducidas (Dietler y Herbich, 1998; Stark, 1998; entre otros). En lo que refiere a la evaluación de las hipótesis formuladas desde los microrrestos respecto a su uso, concretaremos análisis (ver supra) que permitan aproximarnos a la naturaleza del líquido que contuvo (¿agua u otro derivado del procesamiento de algún recurso aún no identificado?) y reconocer la presencia de sustancias no evidentes a partir de los microfósiles. Por otro lado, avanzaremos en describir los hábitos de la gran diversidad de especies y/o géneros de diatomeas identificadas para, considerando información diatomológica microrregional (Grana, Tchilinguirian, Olivera, Laprida y Maidana, 2016), acercarnos a los cuerpos de agua de los que procedieron. Asimismo, se concretarán análisis múltiple de microfósiles de las muestras del interior de la vasija que aún no pudieron ser analizadas por razones presupuestarias (ver supra), así 
como de otras a obtener en el exterior del Recinto 1 Este B. Respecto a los atributos tecnológicos del recipiente, realizaremos análisis de pasta, los que brindarán información sobre las materias primas empleadas y otras técnicas aplicadas en su elaboración a fin de progresar en comprender las prácticas tecnológicas reproducidas por quienes lo crearon. Finalmente, avanzaremos en el estudio de distintos materiales contextualmente asociados para aprehender así el ensamble de prácticas materiales que dieron lugar al contexto arqueológico específico del que fue rescatada.

Sin desconocer los estudios que restan concretar, evaluamos que el análisis de microfósiles y el preliminar acercamiento técnico-morfológico realizado generaron valiosa información y propuestas alentadoras acerca de las prácticas de uso y manufactura asociadas a la primera vasija completa relevada estratigráficamente en LA y posibilitaron una inicial comparación de estas con las involucradas en la elaboración y utilización de otras piezas análogas de la microrregión. De este modo, constituyen un nuevo paso en nuestro objetivo de documentar, considerando contextos diversos y múltiples escalas de análisis, la variabilidad diacrónica y sincrónica de las prácticas materiales (sensu Pauketat, 2001) reproducidas por quienes habitaron el gran conglomerado del fondo de cuenca en la creación y uso cotidiano de su cultura material, para su comparación, en términos cronológicos más exhaustivos, con las de los habitantes de PCa (Elías, 2018) y otras ocupaciones microrregionales anteriores y contemporáneas. En sus prácticas de todos los días los residentes de distintos espacios de ANS reprodujeron, negociaron y transformaron sus límites y relaciones sociales, políticas, económicas, culturales e identitarias (Pauketat, 2001; Stark, 1998). A razón de esto, concluimos señalando que lo expuesto en este trabajo será valioso en términos de continuar progresando en la comprensión del complejo y particular proceso social, cultural, político y económico por ellos conformado luego de ca. 1100 años AP.

Ciudad Autónoma de Buenos Aires, 28 de abril de 2021

\section{Agradecimientos}

El trabajo fue concretado con la financiación y respondiendo a objetivos del Proyecto CIC-CONICET "Prácticas tecnológicas líticas en sociedades del Período Tardío de Antofagasta de la Sierra (Provincia de Catamarca, Puna Meridional Argentina)" (Elías 2018), integrado al Proyecto Arqueológico Antofagasta de la Sierra. Aunque lo mencionado es total responsabilidad de los autores, agradecemos los aportes de los pares revisores que contribuyeron definitivamente a mejorar texto.

\section{Bibliografía}

Babot, M. del P. (2004). Tecnología y utilización de artefactos de molienda en el Noroeste Prehispánico (tesis Doctoral). Universidad Nacional de Tucumán, San Miguel de Tucumán, Argentina.

Balfet, H., Fauvet, M. y Monzón, S. (1992). Normas para la descripción de vasijas cerámicas. CEMCA.

Bertoldi de Pomar, Hetty. (1975). Los silicofitolitos: sinopsis de su conocimiento. Darwiniana, 19 (2-4), 173-206.

Binford, M., Brenner, M. y Leyden, B. (1996). Paleoecology and Tiwanaku Agroecosystems. En A. Kolata (Ed.), Tiwanaku and its hinterlands. ecology and paleoecology of an Andean civilization (pp. 89-108). Smithsonian Institution Press.

Bozarth, S. (1992). Classification of opal phytoliths formed in selected dicotyledons native to the Great Plains. En G. Rapp y S.C. Mulholland (Eds.), Phytolith Systematics (pp. 193-214). Plenum Press.

Cabrera, A. (1976). Regiones fitogeográficas argentinas. En W. F. Kugler (Ed.), Enciclopedia Argentina de Agricultura y Jardinería (2 ${ }^{\circ}$ ed., Tomo II, pp. 1-85). Editorial Acme.

Capparelli, A. y Lema, V. (2010). Prácticas postcolecta/ post-aprovisionamiento de recursos vegetales: una perspectiva paleoetnobotánica integradora aplicada a casas de Argentina. En J. Bárcena y H. Chiavazza (Eds.), Actas del XVII Congreso Nacional de Arqueología Argentina (pp. 1171-1176). Universidad Nacional de Cuyo.

Cohen, L. (2005). Entre guano y arena... Ocupaciones recurrentes: un caso de estudio en el sitio Punta de la Peña 9-III Antofagasta de la Sierra Catamarca (tesis de licenciatura). Universidad Nacional de Tucumán, San Miguel de Tucumán, Argentina.

Cohen, L. (2014). Miradas desde y hacia los lugares de poder. Antofagasta de la Sierra entre 1000-1500 años d.C. Arqueología, 20 (1), 47-72.

Coil, J., Korstanje, A., Archer, S. y Hastorf, C. (2003). Laboratory goals and considerations for multiple microfossil extraction in archaeology. Journal of Archaeological Science, 30, 991-1008.

Elías, A. (2010). Estrategias tecnológicas y variabilidad de los conjuntos líticos de las sociedades tardías en Antofagasta de la Sierra (Provincia de Catamarca, Puna meridional argentina) (tesis Doctoral). Universidad de Buenos Aires, Buenos Aires, Argentina.

Elías, A. (2018). Plan de trabajo y avances presentados para Promoción CIC-CONICET. MS. 
Elías, A. y Escola, P. (2018). Prácticas tecnológicas líticas entre los habitantes de la quebrada de Miriguaca en el escenario sociopolítico tardío de Antofagasta de la Sierra (Puna meridional argentina). Relaciones de la Sociedad Argentina de Antropología, 43 (1), 13-33.

Elías, A., Marconetto, B. y Olivera, D. (2015). Aportes al registro de tarabitas en Antofagasta de la Sierra. Revista de Antropología del Museo de Entre Ríos, 1 (1), 99-102.

Escola, P., Elías, A., Gasparotti, L. y Sentinelli, N. (2015). Quebrada del río Miriguaca (Antofagasta de la Sierra, Puna meridional argentina): nuevos resultados de recientes prospecciones. Intersecciones en Antropología, $16(2), 383-396$.

Fredlund, G. y Tieszen, L. (1994). Modern phytolith assemblages from the North American Great Plains. Journal of Biogeography, 21, 312-335.

Gasparotti, L. y Haros, C. (2015). Contenedores y contenidos. Explorando el consumo de vegetales durante el Período Tardío (Antofagasta de la Sierra, Provincia de Catamarca). Revista del Museo de Antropología, 8 (2), 47-62.

Grana, L., Tchilinguirian, P., Olivera, D., Laprida, C. y Maidana, N. (2016). Síntesis paleoambiental en Antofagasta de la Sierra: heterogeneidad ambiental y ocupaciones humanas en los últimos 7200 años cal AP. Intersecciones en Antropología, 17, 19-32.

Killian, V., Grant, J., Escola, P., Panarello, H. y Olivera, D. (2016). Análisis paleodietario en zonas áridas a través de isótopos estables: el caso de Antofagasta de la Sierra (Noroeste Argentino). Revista Colombiana de Antropología, 52, 199-227.

Korstanje, A. (2005). La organización del trabajo en torno a la producción de alimentos en sociedades formativas (Provincia de Catamarca, República Argentina). (tesis Doctoral). Universidad Nacional de Tucumán, San Miguel de Tucumán, Argentina.

Korstanje, A. y Babot, M. del P. (2007). A microfossils characterization from south Andean economic plants. En M. Madella y D. Zurro (Eds.), Plants, People, and Places: Recent Studies in Phytolith Analysis (pp. 41-72). Oxbow Books.

Maloberti, M. (2019). El paisaje agrario y las prácticas campesinas en el valle de El Bolsón (departamento Belén, Catamarca). Cambios y continuidades en la larga duración (tesis Doctoral). Universidad Nacional de Córdoba, Córdoba, Argentina.

Maloberti, M., Quesada, M., Sastre, J., Amaya, E., Flores, O., Rando, M., Moyano, G. y Vega, M. (2018). Residuos microbiológicos en recipientes cerámicos provenientes de un contexto doméstico. Valle El Bolsón, Departamento Belén, Catamarca (ca. 500 a 300 AP). En Arqueometría 2018. Libro de resúmenes extendidos, Serie Monográfica y Didáctica 56 (pp. 57-61). Universidad Nacional de Tucumán.

Martel, A. y Aschero, A. (2007). Pastores en acción: imposición iconográfica vs. autonomía temática. En A. Nielsen, M.C. Rivolta, V. Seldes, M. Vázquez y P. Mercolli (Eds.), Producción y Circulación Prehispánicas de Bienes en el Sur Andino, (pp. 329-349). Editorial Brujas.

Oliszewski, N. y Olivera, D. (2009). Variabilidad racial de macrorrestos arqueológicos de Zea mays (poaceae) y sus relaciones con el proceso agropastoril en la Puna meridional argentina (Antofagasta de la Sierra, Catamarca). Darwiniana, 47 (1), 76-91.

Olivera, D. y Vigliani, S. (2000/2002). Proceso cultural, uso del espacio y producción agrícola en la Puna Meridional Argentina. Cuadernos del Instituto Nacional de Antropología y Pensamiento Latinoamericano, 19, 459-481.

Olivera, D., Elías, A., Salminci, P., Tchilinguirian, P., Grana, L., Grant, J. y Miranda, P. (2008). Nuevas evidencias del proceso sociocultural en Antofagasta de la Sierra. Informe de campaña año 2007. La Zaranda de Ideas, 4, 119-140.

Parr, Jeff. 2006. Effect of fire on phytolith coloration. Geoarchaeology: An International Journal 21 (2): 171-185.

Pauketat, T. (2001). Practice and history in archaeology: an emerging paradigm. Anthropological Theory, 1 (1), 73-98.

Pearsall, D. (2000 [1989]). Paleoethnobotany: A Handbook of Procedures. Academic Press.

Pérez, M. y Gasparotti, L. (2016). Caracterización petrográfica de las pastas cerámicas de Antofagasta de la Sierra, un enfoque comparativo a nivel intersitios (Puna Austral Argentina). Comechingonia, 20 (1), 175-202.

Piperno, D. (1988). Phytolith Analysis. An Archaeological and Geological Perspective. Academic Press.

Piperno, D. (2006). Phytoliths: A Comprehensive Guide for Archaeologists and Paleoecologists. Altamira Press.

Puente, V. (2020). Informe: descripción morfológica y tecnológica de la vasija rescatada en el Recinto 1 Este de La Alumbrera. MS.

Puente, V., Plá, R. y Invernizzi, R. (2017). La cerámica local de la quebrada del río Las Pitas (Catamarca). Aportes a la 
circulación de personas, saberes y objetos en Antofagasta de la Sierra durante el Tardío. Relaciones de la Sociedad Argentina de Antropología, 42 (1), 35-61.

Salminci, P. (2015). Simetría y diferenciación espacial Los edificios de La Alumbrera. Antofagasta de la Sierra. Arqueología, 21 (1), 89-114.

Shepard, A. (1956). Ceramics for The Archaeologist. Carnegie Institution of Washington.

Stark, M. (1998). Technical choices and social boundaries in material culture patterning: an introduction. En M. Stark (Ed.), The Archaeology of Social Boundaries (pp. 1-11). Smithsonian Institution Press.

Twiss, P. (1992). Predicted world distribution of C3 and C4 grass phytoliths. En G. Rapp y S. C. Mulholland (eds.), Phytolith Systematics (pp. 113-128). Plenum Press.

Zucol, A. (1996). Microfitolitos de las Poaceae argentinas: I: Microfitolitos foliares de algunas especies del género Stipa (Stipae Arundinoideae), de la provincia de Entre Ríos. Darwiniana, 34 (1-4), 151-172. 